\title{
THIRTY-NINE PERFECT NUMBERS AND THEIR DIVISORS
}

\author{
SYED ASADULLA \\ Denartment of Mathematics and Computing Sciences \\ St. Francis Xavier University \\ Antigonish, Nova Scotia. B2G 1CO. Canada \\ (Received July 18, 1984)
}

ABSTRACT. The following results concerning even perfect numbers and their divisors are proved: (1) A positive integer $n$ of the form $2^{p-1}\left(2^{p}-1\right)$, where $2^{p}-1$ is prime, is a perfect number; (2) every even perfect number is a triangular number; (3) $\tau(n)=2 p$, where $\tau(n)$ is the number of positive divisors of $n$; (4) the product of the positive divisors of $n$ is $n^{p}$; and (5) the sum of the reciprocals of the positive divisors of $n$ is 2. Values of $p$ for which 30 even perfect numbers have been found so far are also given.

KEY WORDS AND PHRASES. Perfect number; Marsenne prime; Triangular number. 1980 MATHEMATICS SUBJECT CLASSIFICATION CODE. IOA4O.

1. INTRODUCTION.

A positive integer $n$ is called a perfect number if $\sigma(n)=2 n$, where $\sigma(n)$ is the sum of the positive divisors of $n$. The last digit of the first five perfect numbers $(6,28,496,8128$, and 33550 336) alternates between 6 and 8 . This pattern does not continue as the next three perfect numbers are 8589869056,137 438691328 and 2305843008139952 128. However, it has been proved in [1] that an even perfect number ends in 6 or 28. It is interesting to observe that these are the first two perfect numbers.

2. EVEN PERFECT NUMBERS.

It is well known that positive integers $n$ of the form $2^{p-1}\left(2^{p}-1\right)$, where $2^{\mathrm{P}}-1$ is prime, are perfect numbers. This can be proved using a theorem from elementary number theory [2] which states that if

$n=\prod_{i=1}^{k} p_{i}^{a_{i}}$, where the $p_{i}$ 's are distinct primes and the $\alpha_{i}$ 's are positive integers, then

$$
\begin{aligned}
& \sigma(n)=\prod_{i=1}^{k} \frac{p_{i}{ }^{{ }^{\alpha}+1}-1}{p_{i}-1} . \\
& \text { If } n=2^{p-1}\left(2^{p}-1\right)=2^{p-1} \cdot q \text {, where } q=2^{p}-1 \text { is prime, it follows from }
\end{aligned}
$$
the above theorem that 


$$
\sigma(n)=\frac{2^{p}-1}{2-1} \cdot \frac{q^{2}-1}{q-1}=\left(2^{p}-1\right)(q+1)=\left(2^{p}-1\right) \cdot 2^{p}=2 n \text {, }
$$

which proves that $n$ is a perfect number.

It has been proved in [2] that an even perfect number is of the form $2^{p-1}\left(2^{p}-1\right)$, where $2^{p}-1$ is prime. It can be easily shown that $p$ is prime whenever $2^{P}-1$ is prime, but the converse is false $\left(2^{11}-1=23.89\right.$ is not prime). Primes of the form $2^{p}-1$ are called Mersenne primes.

Thirty-nine even perfect numbers have been found so far [2 - 3] corresponding to $p=2,3,5,7,13,17,19,31,61,89,107,127,521,607,1279,2203,2281,3217$, 4253, 4423, 9689, 9941, 11213, 19937, 21701, 23209, 44497, 86243, 132049, and 216091. No odd perfect number has yet been discovered.

3. EVEN PERFECT NUMBERS AND TRIANGULAR NUMBERS.

The $k^{\text {th }}$ triangular number is defined as

$T_{k}=\sum_{i=1}^{k} i=\frac{1}{2} k(k+1)$. Every even perfect number is a triangular number.

This is proved by noting that

$n=2^{p-1}\left(2^{P}-1\right)=\frac{1}{2}\left(2^{P}-1\right) \cdot 2^{P}=\frac{1}{2} k(k+1)=T_{k}$, where $k=2^{P}-1$.

4. DIVISORS OF AN EVEN PERFECT NUMBER.

If $n$ is an even perfect number, then $\tau(n)=2 p$, where $\tau(n)$ is the number of positive divisors of $n$. This can be easily proved using a theorem from elementary number theory [2] which states that if

$n=\prod_{i=1}^{k} p_{i}{ }^{\alpha_{i}}$, where the $p_{i}$ 's are distinct primes and the $\alpha_{i}$ 's are positive integers, then $\tau(n)=\underset{i=1}{k}\left(\alpha_{i}+1\right)$

The product of the positive divisors of an even perfect number $n$ is $n^{p}$. This is obtained by using still another result from elementary number theory [1], namely,

$$
\underset{i=1}{\tau(n)} d_{i}=n^{\frac{1}{2} \tau(n)} \text {, and the value of } \tau(n)=2 p \text {, }
$$

where the $d_{j}$ 's are positive divisors of $n$.

Finally, the sum of the reciprocals of the positive divisors of an even perfect number is 2. This follows from

$$
\sum_{i=1}^{\tau(n)} \frac{1}{d_{i}}=\frac{(n)}{n} \text {, where the } d_{i} \text { 's are positive divisors of } n \text {, a result }
$$
from elementary number theory [1].

\section{REFERENCES}

1. BURTON, D.M. Elementary Number Theory, Allyn and Bacon, Boston-London-Sydney, 1976.

2. Rosen, K. H. Elementary Number Theory and its Applications, Addison-Wesley, Reading, Massachusetts, 1984.

3. Editorial Panel, Math. Teacher, 79 (1986), p 10. 


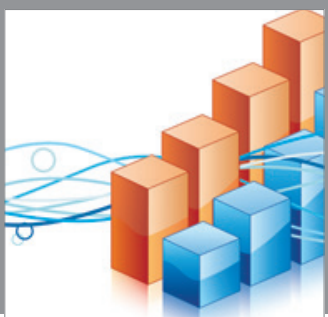

Advances in

Operations Research

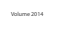

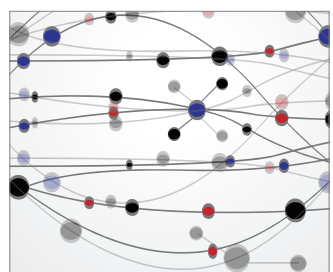

\section{The Scientific} World Journal
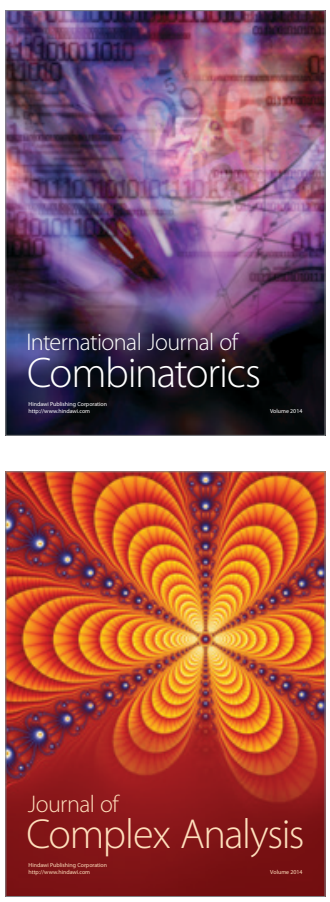

International Journal of

Mathematics and

Mathematical

Sciences
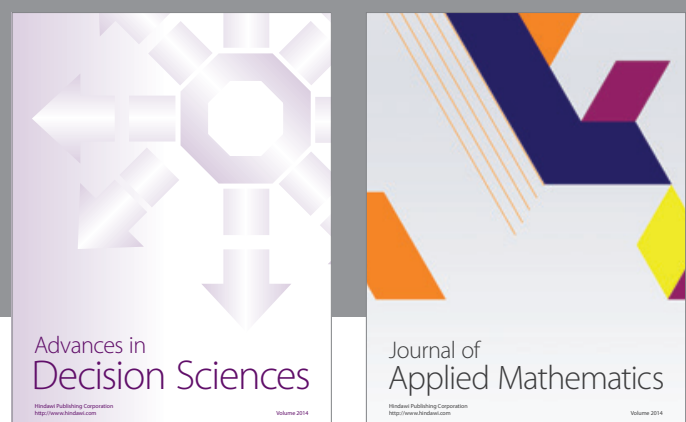

Journal of

Applied Mathematics
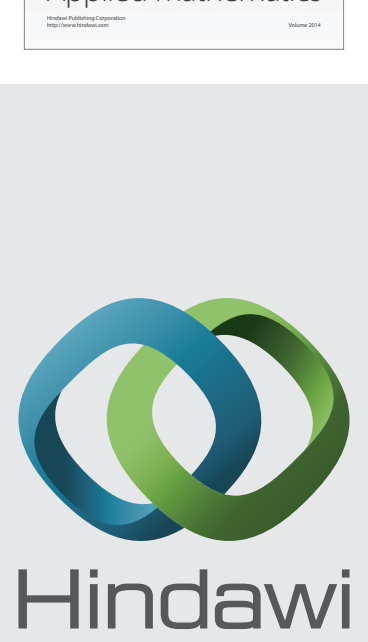

Submit your manuscripts at http://www.hindawi.com
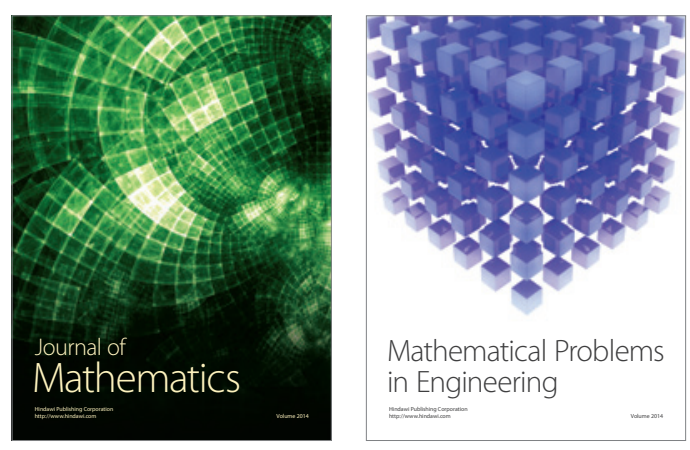

Mathematical Problems in Engineering
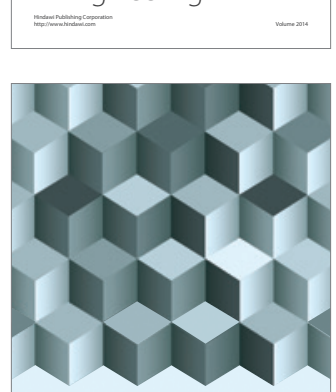

Journal of

Function Spaces
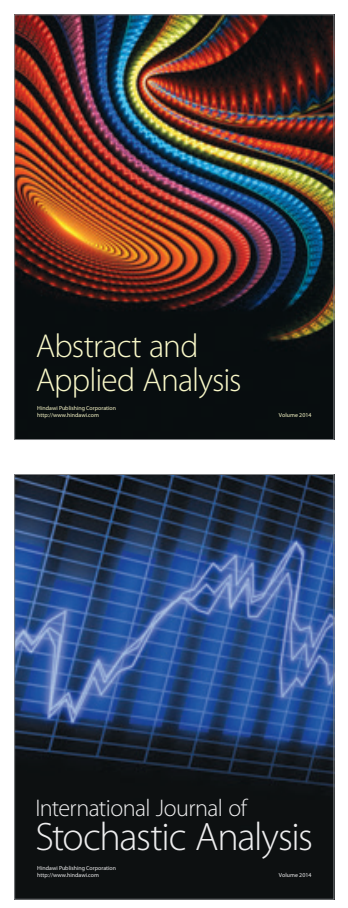

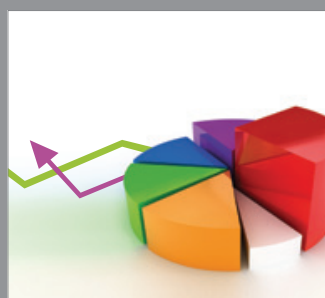

ournal of

Probability and Statistics

Promensencen
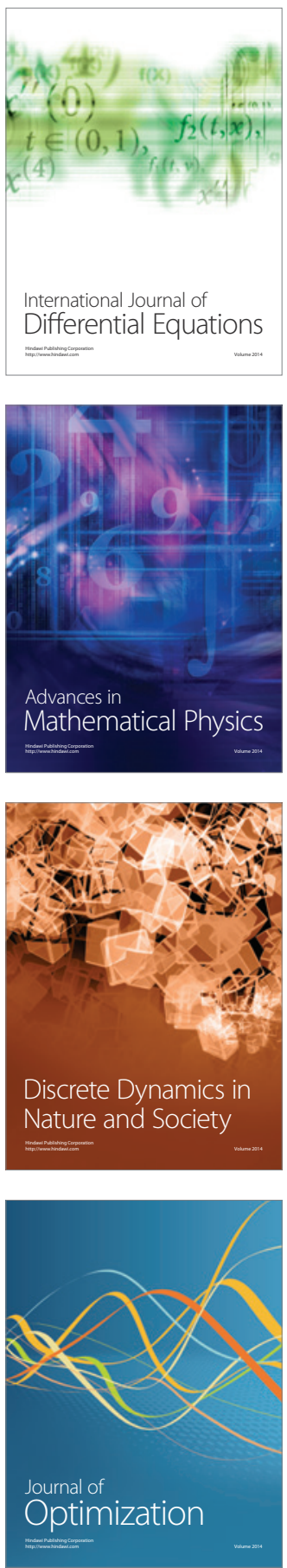